\title{
Annual scientific meeting 27 November 2017
}

\section{SUBTHALAMIC NUCLEUS ACTIVITY \\ PROACTIVELY INFLUENCES RISK ATTITUDE \\ IN PARKINSON'S DISEASE PATIENTS \\ AFFECTED BY PATHOLOGICAL GAMBLING}

\section{A. Mazzoni, ${ }^{1}$ M. Rosa, ${ }^{2}$ J. Carpaneto, ${ }^{1}$ L.M. Romito, ${ }^{3}$ A. Priori, ${ }^{4}$ S. Micera ${ }^{1,5}$}

${ }^{1}$ Translational Neural Engineering Area, Biorobotics Institute, Scuola Superiore Sant'Anna, Pontedera, Italy; ${ }^{2}$ Clinical Center for Neurostimulation, Neurotechnology and Movement Disorders Fondazione IRCCS Ca' Granda Ospedale Maggiore Policlinico, Milan, Italy; ${ }^{3}$ Movement Disorders Department, Neurological Institute Carlo Besta, Milan, Italy; ${ }^{4}$ Aldo Ravelli Research Center for Neurotechnology and Experimental Brain Therapeutics, Department of Health Sciences, University of Milan Medical School, Milan, Italy; ${ }^{5}$ Bertarelli Foundation Chair in Translational Neuroengineering, Institute of Bioengineering and Center for Neuroprosthetics, School of Engineering, Ecole Polytechnique Fédérale De Lausanne, Switzerland

Parkinson's Disease (PD) is a neurodegenerative disorder which significantly affects motor and cognitive functions of the subjects affected, worsening the quality of life of the patients and their next of kin. Therapies alleviating PD motor dysfunctions such as Deep Brain Stimulation (DBS) and assumption of dopaminergic drugs, while improving motor symptoms of PD have been also associated to an increase in impulse control disorders (ICD). It is then of pivotal importance to elucidate the specific dynamics of ICD in PD in order to develop therapies without this critical side-effect.
DBS studies proved that the Sub Thalamic Nucleus (STN) is involved in the dysfunctions in motor and cognitive inhibition in PD patients. However, the vast majority of these studies dealt with reactive and global inhibition so it is still unclear if, how, and to which extent the STN influences the behavior proactively. In the present study the STN activity of twelve PD patients about to start DBS therapy was recorded while the patients performed an economic task in which they were asked to decide between risky (R) and non-risky (NR) options presented on a screen. Half of the patients were previously classified as Pathological Gamblers (PG), an ICD affecting $\sim 5 \%$ of PD patients. As expected, PG patients opted less often than non-PG for NR choices. Analyzing the reaction times of both kinds of patients in different conditions, we found evidence for a proactive determination of this bias. Moreover, PG behavior proved to be strongly affected by the outcome of the previous choice. Then, we analyzed the STN activity in between the display of the outcome of a given choice and the onset of the next cue, looking for neural correlates of the proactive behavioral bias. We found that NR decisions in PG patients were reliably preceded by specific patterns of activation of STN in the seconds before the options presentation onset. This suggests that in PG patients STN activation is needed to achieve an efficient NR choice. No such activation window was found for non-PG patients. In PG patients, the lack of STN previous activation results in a majority of inefficient $\mathrm{R}$ choices, while when STN activates the NR option is predominant in following choices. This suggests that strong STN activation tends to proactively prevent risky choices to counteract urge toward unfavorable choices. The conflict between our impulses and our logic is a basic aspect of human life. Our results suggest that STN participates to this conflict by proactively inhibiting pathological risk drive.

Key words: Parkinson's disease; pathological gambling; subthalamic nucleus.

\section{THE ASTROCYTIC ADAPTOR PROTEIN SHCC/RAI AS A NOVEL TARGET FOR THE THERAPY OF MULTIPLE SCLEROSIS: RESULTS FROM THE EXPERIMENTAL AUTOIMMUNE ENCEPHALOMYELITIS MOUSE MODEL}

\section{Ulivieri, ${ }^{1}$ D. De Tommaso, ${ }^{1}$ F. Finetti, ${ }^{1}$ B. Ortensi, ${ }^{2}$ G. Pelicci, ${ }^{2}$ M.M. D'Elios, ${ }^{3}$ C. Ballerini, ${ }^{4}$ C.T. Baldari ${ }^{1}$}

${ }^{1}$ Department of Life Sciences, University of Siena; ${ }^{2}$ Department of Experimental Oncology, European Institute of Oncology, Milan; Department of Translational Medicine, Piemonte Orientale University "Amedeo Avogadro" Novara; ${ }^{3}$ Department of Experimental and Clinical Medicine University of Florence, Largo Brambilla; ${ }^{4}$ Department of Neurosciences, Viale Pieraccini, University of Florence, Italy

Multiple sclerosis (MS) is an autoimmune disease where myelin-specific $\mathrm{T}$ cells that infiltrate into the central nervous system (CNS) are responsible for the neuroinflammatory reac- tion which ultimately leads to myelin destruction and axonal damage. Recent data indicate however, that the local CNS microenvironment, which is controlled by both infiltrated autoreactive T cells and CNS resident cells, plays a crucial role in MS disease onset and progression. Among CNS resident cells, astrocytes actively modulate the $\mathrm{T}$ cell dependent autoimmune response in MS, however the molecular mechanisms underlying this function are as yet poorly defined. In this context, we have recently demonstrated that ShcC/Rai is as a novel astrocytic adaptor whose loss in mice accounts for a milder experimental autoimmune encephalomyelitis (EAE) in the face of a higher frequency of CNS infiltrated myelin-specific T cells, highlighting the key role played by astrocytes in T cell modulation during EAE and identifying astrocytic Rai as a potential molecular target for MS therapy. Moreover, we have found that Rai is capable to sustain the function of myelin-specific T cells by promoting the astrocyte-mediated production of pro-inflammatory molecules such as IL- 6 and nitric oxide and by maintaining elevated concentrations of extracellular ATP in astrocytes through the inhibition of ATP-degrading enzymes. Taken together, the data suggest that inhibition of Rai in astrocytes during MS may contribute to suppress the activation of myelin-specific $\mathrm{T}$ cells by establishing an anti-inflammatory and immunosuppressive microenvironment.

Key words: ShcC/Rai; astrocytes; EAE; autoimmunity. 


\section{Annual scientific meeting 27 November 2017}

\section{SYSTEMS OF EPILEPTIC SEIZURES PREDICTION: PRELIMINARY DATA}

\section{Insana, ${ }^{1}$ L. de Garazi Zabalo Manrique, ${ }^{2}$ D. Marino, ${ }^{I}$ R. Bruni, ${ }^{I}$ E. Testani, ${ }^{1}$ F. Sarnari, ${ }^{3}$ R. Rocchi, ${ }^{1}$ M. Pranzo, ${ }^{2}$ G. Vatti, ${ }^{1}$ A. Rossi, ${ }^{I}$ P. Detti ${ }^{1}$}

${ }^{1}$ Department of Medical and Surgery Sciences and Neurosciences, University of Siena; ${ }^{2}$ Department of Information Engineering and Mathematics, University of Siena;

${ }^{3}$ Department of Phisics "Enrico Fermi", University of Pisa, Italy

\section{Introduction}

Epilepsy is one of the most common neurological diseases affecting $1 \%$ of the population worldwide. It is characterized by the persistent predisposition to develop epileptic seizures caused by a sudden (often without any kind of warning) and transient hypersynchrous discharge in a pool of cortical neurons. Seizure clinical manifestation depend on the cortical areas of seizure onset and spread. The social burden of epilepsy is very high, patients experience difficulties in achieving education, occupation and often satisfactory relationship. With an appropriate drug treatment seizure can be controlled in a high proportion of patients. Unfortunately, $30 \%$ of them, are drug resistant. A minority of drug resistant patients could benefit of a surgical treatment. Since around 40 years many studies attempted to forecast epileptic seizures through the analysis of biological signals such as ECG and particularly EEG. The research project "PANACEE" (Prevision and Analysis of Brain Activity in Transitions: Epilepsy and Sleep), funded by Regione Toscana, involve the Departments of Neuroscience, Engineering and Mathematics of Siena University and the Departments of Clinical Physiology and Physics of Pisa University. The aim of the project is to de- tect changes in biological signals such as EEG and ECG that could predict an epileptic seizure well in advance to allow an intervention and to implement such measures on wearable devices.

\section{Methods}

We studied 10 patients suffering from drug resistant epilepsy who underwent long term video EEG monitoring as a part of pre-surgical workup. The EEG data has been analyzed to evaluate the degree of phase synchronization between couple of EEG channels across time to estimate a possible change in phase synchronization during the preictal phase. Data were automatically classified using a 1) threshold method specifically developed for the project, and 2) a method based on Support Vector Machine. The analysis has been also performed on data coming from a public EEG database (MIT EEG Database).

\section{Results}

Preliminary results demonstrated a clear cut increase of synchronization measures in some patients. Seizure prediction time ranges from 45 to 90 seconds in most patients. The threshold method is more efficient showing a lower number of false positive "alarms". If confirmed in larger population and in a "real life" setting such results could open a new prospective in the care of drug resistant patients who cannot benefit from surgical treatment.

Key words: seizure; prediction; biological signals.

\section{References:}

Litt B, Echauz J. Prediction of epileptic seizures. Lancet Neurol 2002;1:22-30.

Mormann F, Kreuz T, Andrzejak RG, et al. Epileptic seizures are preceded by a decrease in synchronization. Epilepsy Res 2003:53:173-85.

Moshé SL, Perucca E, Ryvlin P. Tomson T. Epilepsy: new advances. Lancet 2015;385:884 98 .

\section{SLEEP MACROSTRUCTURE AND MICROSTRUCTURE IN PATIENTS WITH DRUG RESISTANT TEMPORAL LOBE EPILEPSY}

\section{E. Testani, A. Giannotta, G. Vatti, L. Insana, D. Marino, B. Pucci, M. Del Testa, R. Rocchi, A. Rossi}

Department of Neurological, Behavioural and Neurosurgical Sciences, Section of Neurological Sciences, University of Siena, Italy

\section{Background}

Sleep and epilepsy are closely related phenomena, for physiopathological and clinical aspects. Both sleep and its deprivation can have an activating effect on epileptic manifestations; on the other hand epileptic seizures may affect the sleep-wake cycle. It has been shown that both critical and intercritical manifestations are linked to different sleep stages; it is now known that sleep NREM, characterized by a 54 high degree of synchrony, has a facilitating action on intercritical and critical anomalies. On the contrary, the desynchronization proper of REM sleep does not represent a fertile ground for epileptic abnormalities [1]. Besides this wellknown dichotomy between non-REM sleep (high activation) and REM sleep (low activation), epileptic phenomena are deeply sensitive to the ongoing level of arousal [2]. In this regard, the study of the Cycling Alternating Pattern (CAP) represent a new reading key permitting to analyze the oscillations of the arousal level. During non-REM sleep the arousal level can be either unstable, as expressed by the repetitive sequences of the CAP, or stable, as reflected by non-CAP. Phase A (arousal complex) and phase B (postarousal rebound response) are the two basic components of the CAP cycle, which presents a $20-40$ s periodicity. Three subtypes of A phases can be recognized: the Al subtypes, which are thoroughly composed of EEG activity with an high degree of synchrony (K-complexes and delta bursts), and subtypes A2 and A3 dominated by moderate (A2) or prominent (A3) EEG desynchrony. CAP can be both a spontaneous endogenous rhythm of sleep, both reactive to perturbations of different nature (exogenous and endogenous) [3]. For this reason, CAP is a useful tool for widening knowledge and studying links between epilepsy and sleep. 


\section{Annual scientific meeting 27 November 2017}

\section{Purposes}

Our purposes are: 1. Compare parameters of sleep macrostructure and microstructure obtained in a population of patients affected by drug resistant temporal lobe epilepsy with those of a control group. 2. Evaluate, in the patient group, the possible correlation of neuropsychological scores that measure memory, depression and anxiety, with sleep microstructure parameters.

\section{Methods}

Ten adult patients affected by drug resistant temporal lobe epilepsy were included in the study (6 males, 4 females, mean age $44.2 \pm 18.0$ years). Each patient was candidate for epilepsy surgery and was hospitalized in the Neurology Department of "Policlinico Santa Maria Le Scotte" in Siena between 2014 and 2017. Each patient underwent prolonged video-EEG monitoring and none of the patients discontinued the anticonvulsant treatment at the time of the study. The patients group was compared with a control group of 10 adult subjects ( 5 males, 5 females, mean age $42.3 \pm 16.7$ years), who underwent polisomnography for sleep study. A visual manual scoring of sleep macrostructure and microstructure was performed (SOMNOmedics software) and macrostructure and CAP parameter were compared between the two groups. In the patients group, a correlation analysis was also performed between microstructural parameters and neuropsychological scales evaluating memory (Digit Span, Course Span, Word Pairs, Rey's Figure, Overlays Courses), depression (Beck Depression Inventory) and Anxiety (State-Trait Anxiety Inventory).

Results

There was a significative difference in REM sleep time (REM time) between the two groups: $68.90 \pm 26.78$ minutes in the patient group and $107.20 \pm 22.03$ in the control group $(\mathrm{P}<0.05)$. Comparison of CAP parameters between the two groups revealed a significative difference in A1 and A2 per- centages between the two groups. The average percentage of Al in the patients group was $73.78 \pm 10.31 \%$, while in controls it was $58.78 \pm 7.35 \%(\mathrm{P}<0.05)$. The average percentage of A2 phases in the patient group was $20.58 \pm 10.26 \%$, while in controls was $32.88 \pm 7.46 \%(\mathrm{P}<0.05)$. Correlation analysis between CAP indexes and neuropsychological scores showed only one significative correlation between CAP rate and the STAI-X2 scale $(\mathrm{r}=0.6382 ; \mathrm{P}=0.047)$.

\section{Conclusions}

Our data indicate that, in patients with drug resistant temporal lobe epilepsy, there are alterations in sleep macrostructure (reduction of REM sleep) and microstructure (increase of $\mathrm{Al}$ and decrease of A2). This data may be the consequence of an alteration in thalamo-cortical circuits due to the damage of mesial temporal structures in patients with drug resistant epilepsy. On the other side, CAP modifications in epileptic patients may represent the consequence of interictal epileptic activity that is not visible in scalp recordings, acting as a disturbing endogenous factor producing reactive CAP. Moreover we found that epileptic patients with high levels of trait anxiety (evaluated by STAI-X2) had an increase in CAP rate; suggesting an alteration of sleep microstructure in those patients.

Key words: epilepsy; CAP; sleep.

\section{References:}

1. Kataria L, Vaughn BV. Sleep and Epilepsy. Sleep Med Clin 2016;11:25-38.

2. Terzano MG, Parrino L, Anelli S,. Halasz P. Modulation of generalized spike-and-wave discharges during sleep by cyclic alternating pattern. Epilepsia 1989;30:772-81.

3. Terzano MG, Mancia D, Salati MR, et al. The cyclic alternating pattern as a physiologic component of normal NREM sleep. Sleep 1985;8:137-45.
ADAPTIVE CELL SURVIVAL PROGRAM IN HYPOXIC NEUROBLASTOMA CELLS: CROSS-TALK BETWEEN HIF-1 $\alpha$ AND THE SHC PROTEIN RAI

\section{Criscuoli, ${ }^{1}$ I. Filippi, ${ }^{1,2}$ D. Osti, ${ }^{3}$ C. Aldinucci, ${ }^{1}$ G. Guerrini, ${ }^{1}$ G. Pelicci, ${ }^{3,4}$ F. Carraro, ${ }^{1,2}$ A. Naldini ${ }^{1}$}

${ }^{1}$ Cellular and Molecular Physiology Unit, Department of Molecular and Developmental Medicine, University of Siena; ${ }^{2}$ Istituto Toscano Tumori, Firenze; ${ }^{3}$ Department of Experimental Oncology, European Institute of Oncology, Milan; ${ }^{4}$ Department of Translational Medicine, Piemonte Orientale University "Amedeo Avogadro", Novara, Italy

Neuroblastoma (NB) is a highly malignant pediatric solid tumor where a hypoxic signature correlates with unfavourable patient outcome. The regulation of oxygen homeostasis is a tightly controlled cellular process, which mainly depends on HIF (hypoxia inducible factor). In particular, HIF- $1 \alpha$ isoform contributes to progression and invasiveness of NB cells. ShC-C/RAI is a neuron-specific isoform of Shc protein, which plays a protective role against cerebral ischemia and it is expressed in several NB cell lines, where it promotes cell survival. In our study, we showed that hypoxia differently affected cell survival and pro-apoptotic program in two NB cell lines, where RAI was or was not expressed respectively SKNBE and SKNMC. The expression of RAI promoted NB cell survival and reduced the expression of proapoptotic markers under hypoxia. Furthermore, we used SKNMC-transfectants overexpressing RAI in order to investigate its role in NB cell responses to hypoxia. Of interest, the overexpression of RAI resulted in higher level of HIF- $1 \alpha$ accumulation under hypoxic condition. This was associated with a) an increased cell survival; b) an increased expression of anti-apoptotic markers; c) a pro-autophagic and not proapoptotic phenotype; d) an increased metabolic activity aimed to promote cell survival. We may conclude that RAI plays an important role in hypoxic signaling in NB cells and the interplay between RAI and HIF- $1 \alpha$ may be relevant in the protection of NB cells against hypoxia. Our results may contribute to further understanding the physiology of NB cells and the molecular mechanisms involved in their survival, with important implications in NB progression.

Key words: hypoxia, neuroblastoma, cell survival, HIF-l $\alpha$, Shc protein. 


\section{Annual scientific meeting 27 November 2017}

\section{INSULIN RECEPTOR-RELATED RECEPTOR: IS THERE A ROLE IN THE LOCAL REGULATION OF PANCREATIC JUICE SECRETION?}

\author{
I.E. Deyev, ${ }^{1}$ M. Regoli, ${ }^{2}$ O.V. Serova, ${ }^{1}$ \\ T.N. Erokhina, ${ }^{1}$ A.A. Mozhaev, ${ }^{1}$ \\ A.G. Petrenko, ${ }^{1}$ E. Bertelli ${ }^{2}$
}

${ }^{1}$ Shemyakin-Ovchinnikov Institute of Bioorganic Chemistry, Russian Academy of Sciences, Moscow, Russia; ${ }^{2}$ Department of Molecular and Developmental Medicine, University of Siena, Italy

The family of the insulin receptor (IR) includes the IR itself, insulin-like growth factor receptor and the insulin receptorrelated receptor (IRR). Whereas the ligands of the first two receptors are known, identification of an IRR ligand has eluded investigations for a long time. For this reason IRR has been considered an orphan receptor. Recently, IRR was found to be activated by mildly alkali media in absence of any protein agonist [1]. IRR shows highly restricted tissue distribution, with the highest level of expression in kidney intercalated cells. Significant amounts of the receptor are also found in the stomach and in the islets of Langerhans. Recent reports indicate that the pancreatic duct system is frequently associated with islet cells. Here, we have been able to demonstrate that islet cells associated with ducts are also IRR-expressing cells. Thus, when the exocrine pancreas is in an active state of secretion duct-associated islet cell behavior is potentially influenced by alkaline juice through IRR activation. To explore this matter, we analyzed the effects of alkaline media a pancreatic $\beta$-cell line (MIN6). IRR-activation was observed and could be inhibited with linsitinib, a synthetic inhibitor of the IR family of receptors. IRR autophosphorylation correlated with $\mathrm{pH}$-dependent linsitinib-sensitive activation of IR substrate 1 (IRS1). In contrast to insulin stimulation, no protein kinase $B$ (Akt/PKB) phosphorylation was detected as a result of the alkali treatment. Alkaline medium, but not insulin, also triggered actin cytoskeleton remodeling in MIN6 cells. Cell pre-incubation with linsitinib prevented actin rearrangement. We propose that the activation of IRR by alkali is a component of a local loop of signaling between the exocrine and endocrine parts of the pancreas aimed to regulate bicarbonate secretion in the pancreatic juice.

Key words: insulin receptor-related receptor; MIN-6 cells; pancreas.

\section{References:}

1. Deyev E, Sohet F, Vassilenko K, et al. Insulin receptor-related receptor as an extracellular alkali sensor. Cell Metab 2011;13:679-89.

\section{THINPAD: AN INTEGRATED EFFORT TO TARGET THE HIV-1 NUCLEOCAPSID PROTEIN}

\section{Mori, M. Botta \\ Department of Biotechnologies, Chemistry and Pharmacy, University of Siena, Italy}

AIDS is one of the most serious pandemic diseases of the modern era. Although current therapies based on targeting key processes of the HIV-1 replication cycle are potent and selective, several clinical failures are recorded due to the emergence of drug resistance. Hence, there is an urgent need for novel drugs and alternative therapeutic strategies. The ob- jective of the THINPAD consortium is to discover and develop novel anti-HIV agents targeting the HIV-1 nucleocapsid protein (NC), which is one of the most conserved sequence within HIV-1 strains and is highly required for HIV-1 replication, being therefore a primary target to overcome antiretroviral drug-resistance.

Within the framework of THINPAD, we recently established a multidisciplinary approach combining in silico screening, fluorescence-based molecular assays and cellular antiviral assays to discover non-covalent NC inhibitors. A number of lead compounds have been identified and profiled up to early preclinical studies, thus becoming promising candidates for further development.

Key words: AIDS/HIV; nucleocapsid protein; drug resistance. 